\title{
Assessment of Conventional Insecticides against Common Bed Bugs Cimex Hemipterus (Hemiptera: Cimicidae) in Jeddah Province, Saudi Arabia
}

\author{
Samar A. Khan', Jazem A. Mahyoub ${ }^{2}$, Khalid M. Al-Ghamdi'2, \\ Samia Q. Alghamdi ${ }^{3 *}$, Tariq S. Alghamdi ${ }^{3}$ and Fatehia N. Gharsan ${ }^{3}$ \\ ${ }^{1}$ Department of Biology, Faculty of Science, University of Jeddah, Saudi Arabia. \\ ${ }^{2}$ Faculty of Science, King Abdulaziz University, Jeddah, Saudi Arabia. \\ ${ }^{3}$ Department of Biology, Faculty of Science, Al-Baha University, Saudi Arabia.
}

http://dx.doi.org/10.13005/bbra/2957

(Received: 05 August 2021; accepted: 02 November 2021)

\begin{abstract}
Bed bugs, Cimex hemipterus (Hemiptera: Cimicidae) are small insects that are considered public health pests and cause many health and economic problems. The efficacy of Organophosphorus insecticides (Safrotin and Actikill) and pyrethroids (Project and Cyper Safe) in controlling $C$. hemipterus adults and nymphs were investigated using contact and dipping methods. The WHO protocol was followed for insecticides application. The results of the current study showed that the project pesticide from the pyrethroids group gave the highest effectiveness against bed bugs, as the value of the concentration that killed $50 \%$ (LC50) of insects was of the adults $(264.8,337.5 \mathrm{ppm})$ when using dipping and contact methods, respectively. while the LC50 value when applying the two methods of the same insecticide against nymphs was 254.7 and 329.3ppm, respectively. However, Safrotin the more effective organophosphorus insecticide. The dipping method was more effective than the contact method for all insecticides, and the nymphs were more susceptible than the adults $(\mathrm{LC50}=1502.6$ and $1065.28 \mathrm{ppm})$. The pesticides can be arranged in descending order according to their effect as follows: Project> Cyper Safe > Safrotin > Actikill. Our findings suggest that bed bugs in Jeddah Province may have developed resistance to common pesticides used in public health pest management programs. For this reason, this study recommends a periodic evaluation of the effectiveness of pesticides to be used during emergency pest outbreaks
\end{abstract}

Keywords: Assessment; Bed Bug; Concentration Insecticide; Contact Method; Dipping Method; Organophosphorus Insecticides; Pyrethroid Insecticides.

Bed bugs, Cimex hemipterus (Hemiptera: Cimicidae) infestation is a serious nuisance in homes, schools, mosques, and shelters as well as recreational and tourist sites leading to economic losses. Bed bug bites can cause a variety of skin lesions, including allergic and cutaneous inflammation. Moreover, bed bugs are vectors of several pathogens. Although in vitro studies have shown that human immunodeficiency virus (HIV) cannot be transmitted through cross-feeding systems $^{1}$, hepatitis B virus (HBV) and HIV can survive in the blood of bed bugs for 4 and $2 \mathrm{~h}$, respectively ${ }^{2,3}$. Ogston et $\mathrm{al}^{4}$ reported that hepatitis $\mathrm{B}$ can survive for up to 6 weeks in the blood of bed bugs fed HBsAg-positive meal. Clinically, bed bugs can successfully transmit numerous diseases, 
including plague, typhoid fever, and viral fever. Moreover, Brittny et $\mathrm{al}^{5}$ noted the presence of bed bugs in areas affected by Chagas disease epidemics and several species of pathogenic fungi, including Trichoderma sp. and Aspergillus sp. has been isolated from the bed bug species $C$. Hemipterus (Fabricius, 1803) ${ }^{6}$.

Over the years, chemical-based insecticides, such as pyrethroids, have been widely used in controlling insect infestation. However, some insect strains, including bed bugs, have developed resistance to some of the commonly used insecticides ${ }^{7-9}$. Bed bugs could be susceptible to organic chlorides, including DDT and organophosphate insecticides; however, indoor use of these insecticides is prohibited in several countries because of their high toxicity ${ }^{10}$. Propoxur, a carbamate insecticide, is an effective alternative against pyrethroid-resistant bugs and is commonly used in controlling bed bug infestation in commercial and industrial buildings, hospitals, and hotels ${ }^{11}$.

Insecticide resistance is caused by genetic mutations, which increase the resistance threshold of insects to commonly used insecticides ${ }^{12}$. Owing to the resistance of insects to some insecticides, routine studies are warranted to examine the effectiveness of common insecticides in controlling bed bugs. Therefore, this study aimed to examine the efficacy of insecticides commonly used in Jeddah Province, Saudi Arabia, for controlling nymphs and adults of $C$. hemipterus, and to compare the effects of contact and dipping methods of application.

\section{MATERIALS AND METHODS}

\section{Bed bug rearing}

Bed bugs (C. hemipterus mother strain) were collected from various neighborhoods in Jeddah and transported in a glass container to the bed bug breeding laboratory in the Dengue and Vector Control Research Unit, Department of Biology, King Abdulaziz University. Pieces of folded white paper were placed inside the bottles containing the bugs to simulate a natural environment and to enable the bugs to move around inside the bottles. The openings of the breeding bottles were covered with white muslin cloth and strapped tightly using rubber bands to prevent the bed bugs from escaping. The cloth was tightened to simulate human skin and facilitate bed bug feeding. The breeding process was performed under a 12-h light and dark photoperiod at $25 \pm 1$ ! and a relative humidity of approximately $60 \% \pm 5 \%$, according to the method described by Feldlaufer et $\mathrm{al}^{13}$ and $\mathrm{Zha}$ et $\mathrm{al}^{14}$. Breeding was performed to obtain a sufficient number of bugs for the insecticide evaluation experiments. To stimulate egg production, the bed bugs were fed rabbit blood for approximately $30 \mathrm{~min}$ once a week. This was accomplished by attaching the cloth-covered opening of each breeding bottle directly onto the skin of a rabbit.

\section{Insecticide compounds tested}

Two organophosphorus insecticides, Safrotin (20\% propetamphos) and Actikill (500 $\mathrm{g} / \mathrm{L}$ pirimiphos-methyl), and two pyrethroid insecticides, Project (5\% alpha-cypermethrin) and Cyper Safe (10\% cypermethrin), were tested in this study.

\section{Insecticide susceptibility tests}

The efficacy of an insecticide against bed bugs is determined based on its active ingredient and the application method. Therefore, in this study, we examined the efficacy of the four insecticides using contact and dipping methods. The contact method simulated residual pesticide application whereas the dipping method simulated residual and non-residual spraying.

The World Health Organization (WHO) was used the standard tube method to examine the efficacy of the insecticides when applied by contact to $C$. hemipterus nymphs and adults. An aspirator was used to introduce 20 -bed bugs into four holding tubes (length: $12.5 \mathrm{~cm}$ and diameter: $4.4 \mathrm{~cm}$ ) containing untreated Whatman No. 1 filter papers. The bugs were then gently blown into the exposure tubes containing Whatman No. 1 filter papers impregnated with the insecticides. After 1-h exposure, the bugs were transferred back into the holding tubes. The number of bugs knocked down at $60 \mathrm{~min}$ and mortalities at $24 \mathrm{~h}$ were recorded following the WHO protocol. One of the tubes containing untreated filter papers was designated as the control group.

The dipping method involved placing the $C$. hemipterus nymphs and adults in small muslin bags and then directly immersing them in the insecticides for $30 \mathrm{~s}$. Thereafter they were 
transferred to $150-\mathrm{mL}$ clean containers, labelled in advance with the name and concentration of the insecticide used. The number of mortalities after $24 \mathrm{~h}$ was recorded.

\section{Statistical analysis}

MS-Excel was used for data validation. Regression analysis was performed to assess the dosage response of the bed bugs to the insecticides. Chi-square slope values, lethal concentration $\left(\mathrm{LC}_{50}\right.$ and $\mathrm{LC}_{90}$ values), fiducial limits, and slopes were determined from the regression curves according to the procedure described by Litchfield and Wilcoxon ${ }^{15}$. Statistical analyses were performed using the method of evaluating biological data.

\section{RESULTS}

\section{Organophosphorus insecticides}

The results of this study are presented in Table 1. These results show that there was a relationship between tested concentrations and mortality percentage of adults and nymphs of C. hemipterus treated with organophosphorus insecticides, with concentrations used ranged from 500 to 5000 (ppm). Mortality rates ranged from $2-78$ and $4-83 \%$ in the dipping method, and
5-56 and 6-66\% in the contact method for adults and nymphs, respectively; furthermore, mortality rates of adults and nymphs treated with Actikill insecticide ranged from 17-77, 21-88, 3-65 and $5-72 \%$ respectively in the dipping and contact method. The study of the toxicity lines LC-P lines (Figure 1), LC50 and LC90for adults insects and nymphs treated with a Safrotin insecticide in both dipping and contact methods demonstrated that there is a remarkable difference in the level of sensitivity.The nymphs were found to be more sensitive than adults by 1.22 and 1.23 during exposure to Safrotin, and 1.72 and 1.21 treated with Actikill insecticides. The optimal result were recorded with a dipping method compared to the contact method with adults (1.42 and 1.82 times); nymphs (1.41 and 1.82 times) respectively with Safrotin and Actikill; as in the case of Safrotin pesticide, also evaluated different concentrations to kill $50 \%$ of adults and nymphs after $24-48$ hours exposure to dipping method (1297.3 and $1065.3 \mathrm{ppm}$ and contact (1844.1 and $1502.6 \mathrm{ppm}$ ) respectively. The results have shown that the concentrations needed to kill $50 \%$ of adults and nymphs after 24-48 hours with Actikill insecticide were 2450.4 and1421.6 ppm and 3128.9 and 2580.9

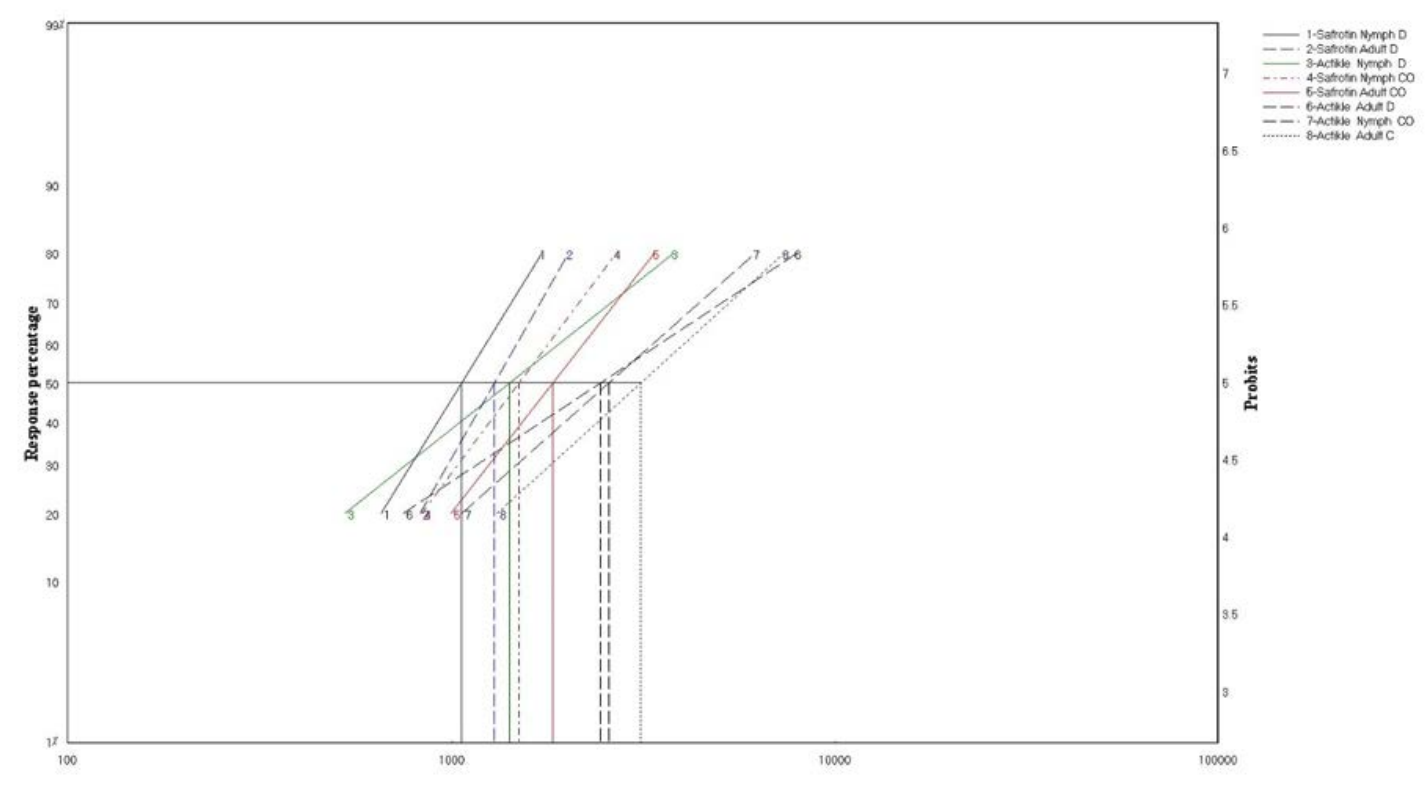

Fig. 1. Regression analysis to assess the toxicity of the organophosphorus insecticides (Safrotin and Actikill) to adult and nymph bed bugs (Cimex hemipterus) in relation to the method of application. ("D" represents "dipping" and that "CO" represents "contact") 
ppm by dipping and contact methods respectively as shown in Table (1). Overall, the results showed that Safrotin was 1.89 and 1.33 times more effective in controlling the adults and nymphs, respectively, than the other organophosphorus insecticide when applied by dipping, and 1.69 and 1.72 times more effective in controlling the adults and nymphs, respectively, using the contact method (Figure 1). Pyrethroid insecticides

The results presented in the Table 2 show the level of sensitivity of adults and nymphs to the pyrenoid insecticides using the dipping and contact methods. The nymphs were found more sensitive than the adults to selected insecticide exposure using the dipping method. The LC50 and LC90 values of the Project insecticide for the adult bed bugs subjected to dipping were 264.8 and 568.9 ppm, respectively, after $24-48 \mathrm{~h}$, whereas the values for those subjected to the contact treatment over the same period of time were 337.5 and 1128.4 ppm, respectively. The LC50 and LC90 values for Project insecticide for the nymphs subjected to the dipping treatment were 254.7 and $706.4 \mathrm{ppm}$, respectively, whereas the values for those subjected to the contact treatment were 329.3 and $667.5 \mathrm{ppm}$, respectively (Table 2).
The adults and nymphs of bed bugs displayed different levels of sensitivity to Cyper Safe insecticide using the dipping and contact treatment methods. Overall, the nymphs were found more sensitive to the insecticide than the adults, and the dipping method was more effective than the contact method. The $\mathrm{LC}_{50}$ and $\mathrm{LC}_{90}$ values of Cyper Safe insecticide for the adult bed bugs subjected to the dipping treatment were 416.7 and $1196.4 \mathrm{ppm}$, respectively, after $24-48 \mathrm{~h}$, whereas the values for those subjected to the contact treatment were 526.9 and $1554.4 \mathrm{ppm}$, respectively. The $\mathrm{LC}_{50}$ and $\mathrm{LC}_{90}$ values of Cyper Safe insecticide for nymphs subjected to the dipping treatment were 369.9 and $764.5 \mathrm{ppm}$, respectively, whereas the values for those subjected to the contact treatment were 474.9 and $1606.7 \mathrm{ppm}$, respectively (Table 2). The resistance of the adult and nymph bed bugs to Cyper Safe insecticide ranged between 1.64 to 2.07times (Figure 2 and Table 2), indicating that Project insecticide was the most effective pyrenoid insecticide and it was found that the dipping method produced the best results (Figure 3).
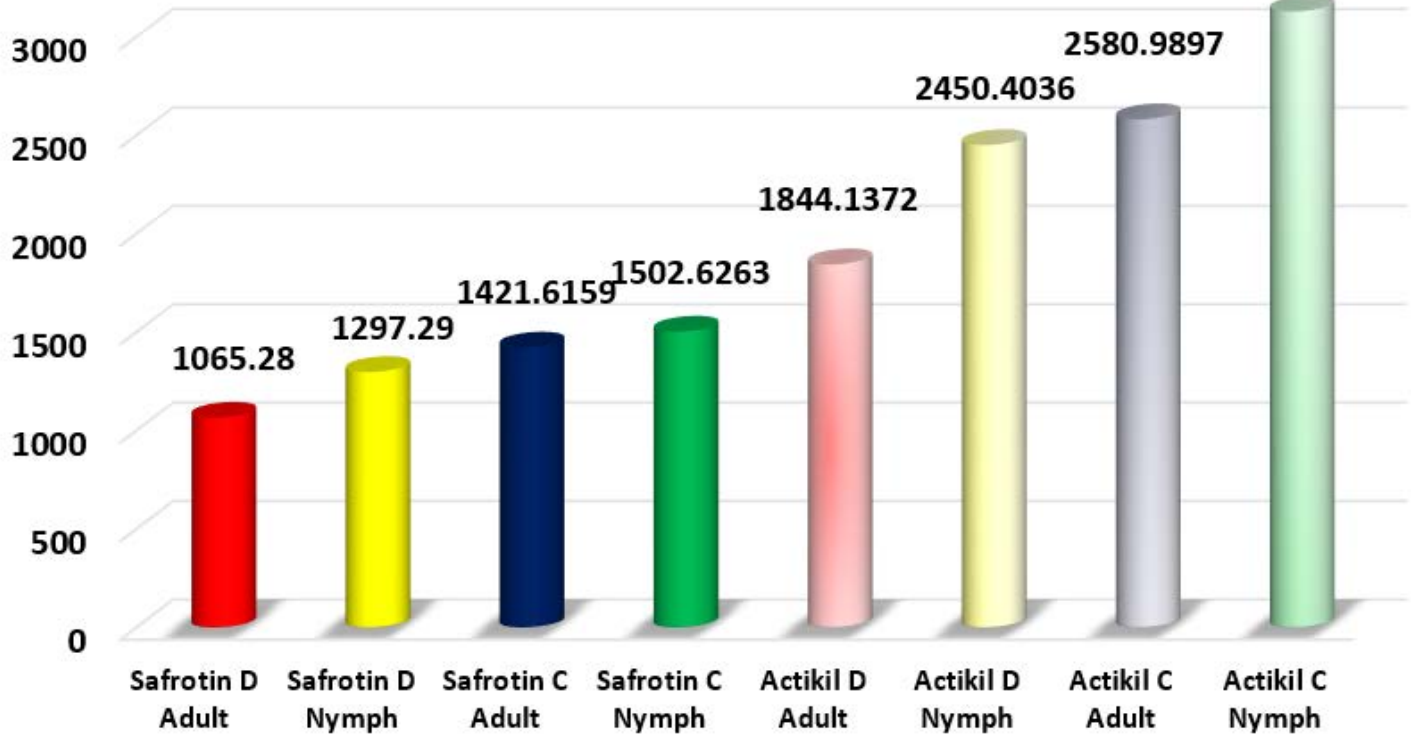

Fig. 2. Toxicity of Safrotin and Actikill to adult and nymph bed bugs (Cimex hemipterus) in relation to the method of application. "D" represents "dipping" and that "CO"” represents "contact." 
KHAN et al., Biosci., Biotech. Res. Asia, Vol. 18(4), 757-765 (2021)

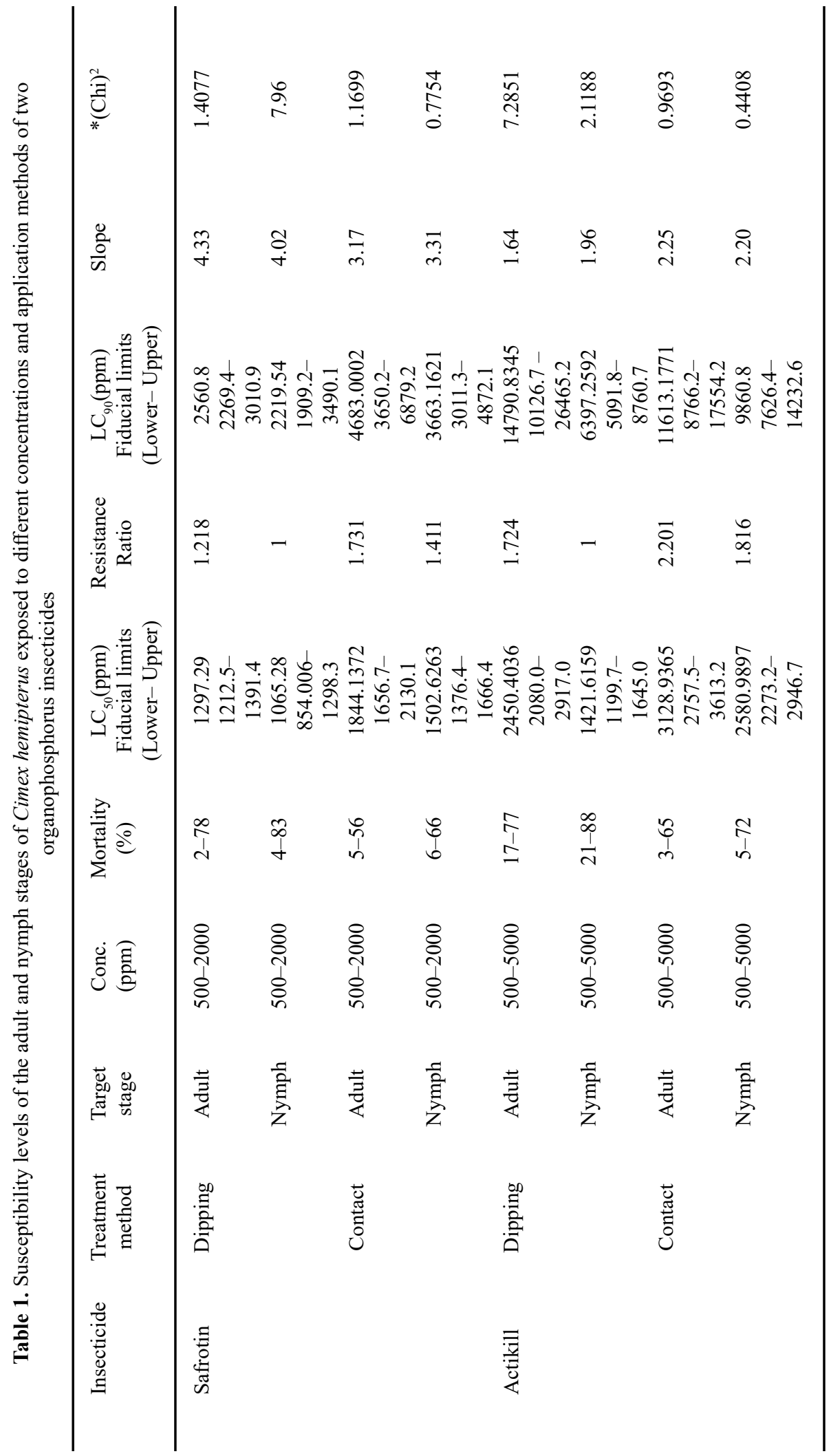




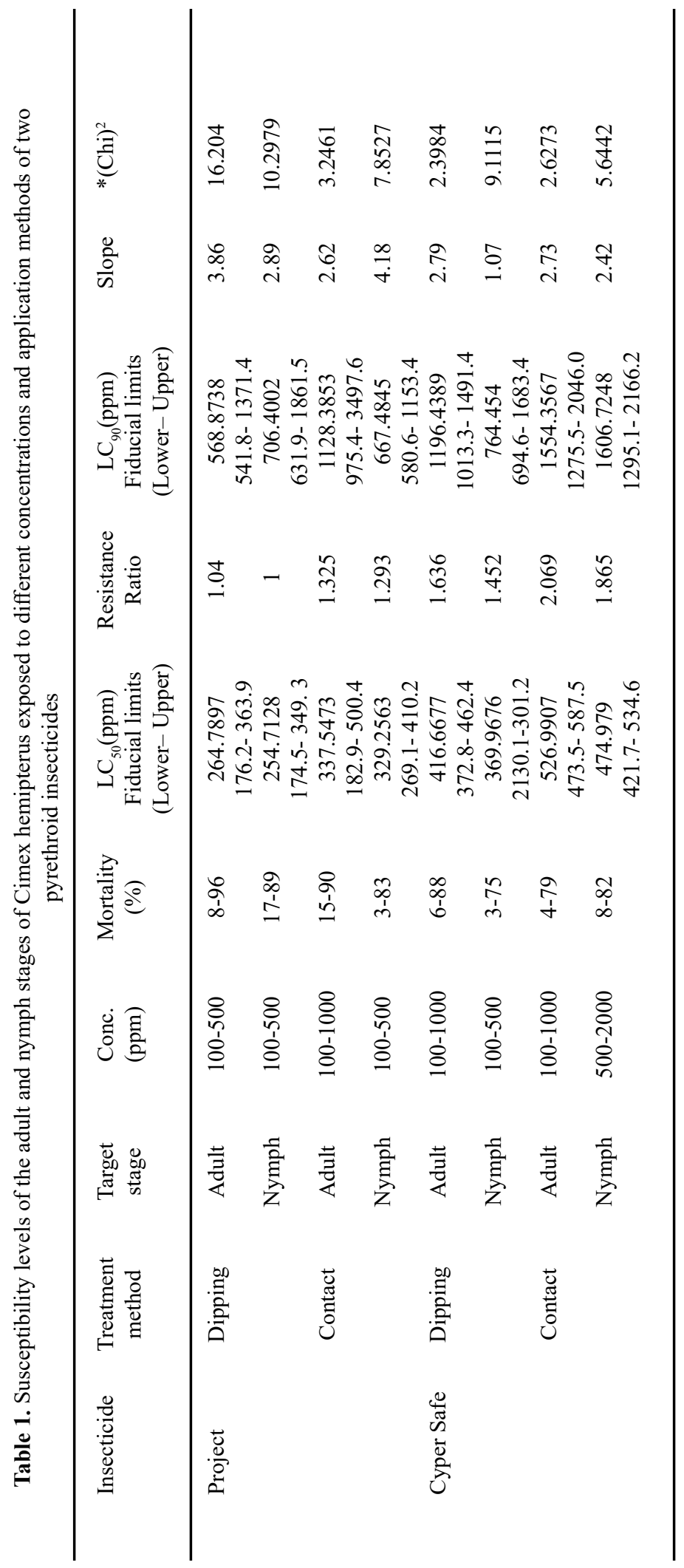




\section{DISCUSSION}

The results of present study have shown that the Safrotin insecticide was more effective than the Actikill insecticide of the same group. The toxicity of organophosphorus insecticides is attributed due to the inhibition of the enzyme acetylcholinesterase, which is responsible for the breakdown of the excitatory neurotransmitter resulting in the stoppage the nerve impulses. This leads to the accumulation of acetylcholine at synapses and caused an increase in excessive excitability and eventually death of the insects ${ }^{16}$. Furthermore, Project was more effective of the two pyrethroid insecticides. The toxicity effect of pyrethroid insecticides may lead to their ability to change the function of voltage-gated sodium channels in the neuron membrane of insects. As a result, this can disturb the electrical signals in the nervous system ${ }^{17}$. Differences in the efficacy of the insecticides may be due to the differences in their active components, exposure periods, and frequency of spraying. In the present study, the nymphs were found more susceptible to insecticides than the adults. This observation may be attributed to the thinner and permeable body wall of the nymphs compared to that of the adults.
Additionally, adults have a larger body surface than nymphs, which may have contributed to the higher susceptibility of the nymphs to the insecticides. The dipping method was more effective with nymphs than the contact method because, through dipping, the body surface of the nymphs and adults was immersed in the insecticides. Nevertheless the comparable regional studies are limited; although numerous studies have been performed in other regions and the results of which are consistent to those of our study. In the present study, relatively high concentrations of the insecticides were used to obtain the desired result, indicating that the nymph and adult bed bugs have developed a certain level of resistance to the insecticides, which are commonly used in insect pest control programs in Jeddah province. Despite the toxic effects of two groups of pesticides, the current study has shown a significant decrease in the response of nymphs and adults of bed bugs to the tested pesticides, and to obtain appropriate mortality rates for drawing toxicity lines and extracting statistical constants, very high concentrations of the tested pesticides were used. This is an indication of the possibility of the bugs acquiring the resistance to the pesticides used in the control programs in Jeddah province, with a difference in the level

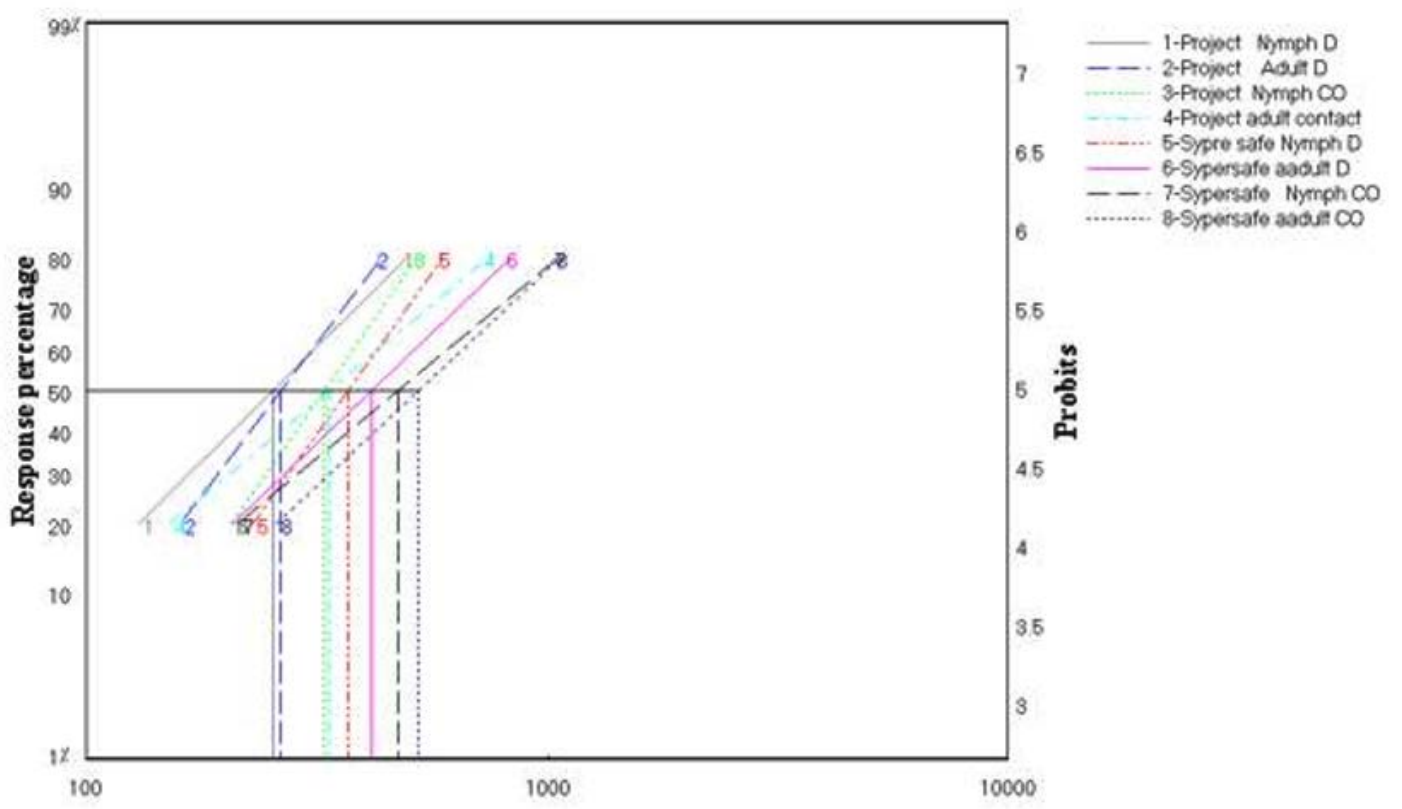

Fig. 3. Toxicity lines IC-p lines that show the different level of sensitivity to bed bugs C.hemipteruswith a different in tested pesticides, target phase and method of application 
of response from tested compounds. Previous studies have reported the acquisition of resistance by insects to commonly used insecticides. Boase et $\mathrm{al}^{18}$ demonstrated that bed bugs in Britain and the United States of America are resistant to the pyrethroid insecticide á-cypermethrin. Similarly, bed bugs in Sydney, Australia are resistant to some pyrethroid insecticides, including deltamethrin ${ }^{19,20}$. Globally, several studies have demonstrated insect resistance to several traditional phosphorous, carbamate, and pyrethroid chemical compounds ${ }^{19-24}$. Furthermore, some previous studies attributed pesticide resistance in bed bugs to behavioral factors, such as avoidance to treated environments. However, Hardstone et $\mathrm{al}^{25}$ reported that pesticide resistance in bed bugs could be attributed to their ability to oxidize toxic substances through cytochrome p450 activity. Benoit et al ${ }^{26}$ reported that insecticide resistance in bugs could be a result of physiological adaptations, as the target sites of the pesticides may become insensitive to the particular pesticides. The reason for insect resistance to phosphorous pesticides due to an increased capacity to metabolism that can be the result of either modification of the existing enzyme to more suitable for degradation of insecticides, or by increasing production of detoxification of enzymes which were previously existing in susceptible insects, yet in much lower amounts ${ }^{27}$. Lastly, this study was conducted in vitro; hence, further studies required to be applied in the fields.

\section{Conclusion and recommendations}

In conclusion, in this study, relatively higher concentrations of the insecticides were needed to achieve the desired level of toxicity against the nymph and adult stages of bed bugs than the concentrations normally used against other insects that impact public health, including mosquitoes, flies, and ticks. This is an indication that bed bugs present in Jeddah province may have acquired a certain level of resistance to the pesticides commonly used in public health pest control programs. Therefore, routine studies are warranted to examine the sensitivity of major insect pests to commonly used pesticides for detecting the emergence of mutant strains. A periodic evaluation of the pesticides by public health should be carried out to be used at the time of the spread of pests, and it will also benefit in the creation of database of pesticides and their effectiveness. The experiments will assist to formulate new concentration of insecticide for killing bed bugs insect. It would prepare insecticed to rate in the write for creating income.Further extensive studies on insecticide efficacy against common bed bugs are really needed.

\section{ACKNOWLEDGEMENTS}

The authors would like to thank the team of researchers at the Dengue Fever and Vector Control Unit, King Abdulaziz University, for their support during this study.

\section{Funding}

This work was not supported by any funding agencies.

\section{Disclosure statement}

No potential conflict of interest was reported by the authors.

\section{Data availability statement}

All data generated or analyzed during this study are included in this manuscript.

\section{REFERENCES}

1. Webb PA, Happ CM, Maupin GO, et al. Potential for insect transmission of HIV: experimental exposure of Cimex hemipterus and Toxorhynchites amboinensis to human immunodeficiency virus. J Infect Dis. 1989;160(6):970-977.

2. Blow JA, Turell MJ, Silverman AL, et al. Stercorarial shedding and transtadial transmission of hepatitis B virus by common bed bugs (Hemiptera: Cimicidae). J Med Entomol. 2001;38(5):694-700.

3. Jupp PG, Lyons SF. Experimental assessment of bed bugs (Cimex lectularius and Cimex hemipterus) and mosquitoes (Aedes agypti formosus) as vectors of human immunodeficiency virus. AIDS. 1987;1(3):171-174.

4. Ogston CW, Wittenstein FS, London WT, et al. Persistence of hepatitis B surface antigen in the bed bug Cimex hemipterus (Fabr.). J Infect Dis. 1979;140(3):411-414.

5. Blakely BN, Hanson SF, Romero A. Survival and transstadial persistence of Trypanosoma cruzi in the bed bug (Hemiptera: Cimicidea), J Med Entomol. 2018;55(3):742-746.

6. Ab Majid, A.H. and Ahmad, A.H. Laboratory rearing of Cimex hemipterus F. (Hemiptera: Cimicidae) feeding on different types of human blood compositions by using modified artificial feeding system. Asian Pacific Journal of Tropical 
Disease, 2015;5(12), pp.930-934.

7. Koganemaru R, Miller DM. The bed bug problem: Past, present, and future control methods. Pestic Biochem Phys. 2013;106(3):177-189.

8. Davies TGE, Field LM, Usherwood PNR, et al. DDT, pyrethrins, pyrethroids and insect sodium channels. IUBMB Life. 2007;59(3):151-162.

9. Potter MF. The history of bed bug managementwith lessons from the past. Am Entomol. 2011;57:14-25.

10. Romero A, Potter MF, Potter DA, et al. Insecticide resistance in the bed bug: a factor in the pest's sudden resurgence? J Med Entomol. 2007;44(2):175-178.

11. EPA, Environmental Protection Agency, Notice of pesticide re-registration treatment, 2013.

12. Dang K, Doggett SL, Veera Singham G, et al. Insecticide resistance and resistance mechanisms in bed bugs, Cimex spp. (Hemiptera: Cimicidae). Parasit Vectors. 2017;10(1):318.

13. Feldlaufer FM, Harlan HJ, Miller DM. Laboratory rearing of bed bugs. In: Karl M, Farida M, editors. Rearing Animal \& Plant Pathogen Vectors, 1(1), pp.119-130.

14. Zha C, Wang C, Li A. Toxicities of selected essential oils, silicon oils, and paraffin oil against the common bed bug (Hemiptera: Cimicidea). $J$ Econ Entomol. 2018;3(1):170-177.

15. Litchfield JT, Wilcoxon F. A simplified method of evaluating dose-effect experiments. J Pharmacol Exp Ther. 1949;96(2):99-113.

16. Siegfried BD, Scharf ME. Mechanisms of organophosphate resistance in insects. InBiochemical sites of insecticide action and resistance. Springer, Berlin, Heidelberg. 2001: pp.269-291.

17. Soderlund DM. Molecular mechanisms of pyrethroid insecticide neurotoxicity: recent advances. Archives of toxicology. 2012;86(2):16581.

18. Boase C, Small G, Naylor R. Interim report on insecticide susceptibility status of UK bed bugs. Professional Pest Controller. 2006:6-7.
19. Moore DJ, Miller DM. Laboratory evaluations of insecticide product efficacy for control of Cimex lectularius. J Econ Entomol. 2006;99(6):20802086.

20. Lilly DG, Zalucki MP, Orton C, et al. Confirmation of insecticide resistance in Cimex lectularius Linnaeus (Hemiptera: Cimicidae) in Australia. Austral Entomol. 2015;54:96-99.

21. Tawatsin A, Thavara U, Chompoosri J, et al. Insecticide resistance in bedbugs in Thailand and laboratory evaluation of insecticides for the control of Cimex hemipterus and Cimex lectularius (Hemiptera: Cimicidae). J Med Entomol. 2011;48(5):1023-1030.

22. Lilly DG, Doggett SL, Zalucki MP, et al. Bed bugs that bite back, confirmation of insecticide resistance in the common bed bug, Cimex lectularius. Prof Pest Manag. 2009;13:22-24.

23. Kilpinen $\mathrm{O}$, Kristensen M, Jensen KMV. Resistance differences between chlorpyrifos and synthetic pyrethroids in Cimex lectularius population from Denmark. Parasitol Res. 2011;109(5):1461-1464.

24. Romero A, Anderson TD. High levels of resistance in the common bed bug, Cimex lectularius (Hemiptera: Cimicidae), to neonicotinoid insecticides. $J$ Med Entomol. 2016;53(3):727-731.

25. Hardstone MC, Strycharz JP, Kim J, et al. Development of multifunctional metabolic synergists to suppress the evolution of resistance against pyrethroids in insects that blood feed on humans. Pest Manag Sci. 2015;76(6):842-849.

26. Benoit JB, Adelman ZN, Reinhardt K, et al. Unique features of a global human ectoparasite identified through sequencing of the bed bug genome. Nat Commun. 2016;7:10165.

27. Siegfried, Blair D., and Michael E. Scharf. "Mechanisms of organophosphate resistance in insects." Biochemical sites of insecticide action and resistance. Springer, Berlin, Heidelberg, 2001; 269-291. 